\title{
LA COHERENCIA DE LA DISCIPLINA DEL DUMPING CON LA DISCIPLINA DE LA LIBRE COMPETENCIA EN EL PERÚ: EL CASO DE DUMPING DE LA REPÚBLICA POPULAR DE CHINA THE COHERENCE OF THE DUMPING AND FREE MARKET POLICIES IN PERU: THE DUMPING CASE OF THE PEOPLE'S REPUBLIC OF CHINA
}

\author{
Geoffrey Cannock ${ }^{*}$ \\ APOYO Consultoría \\ Rodrigo Ezcurra* \\ APOYO Consultoría
}

The Peruvian competition authority Indecopi simultaneously upholds the duties of ensuring a free market as well as protecting it from distortions caused by dumping practices. Such incoherence is evident, since both disciplines are based upon opposite principles: one favors free trade, while the other tends toward protectionism.

In this article, the authors explore Indecopi's dumping policy and how it can collide with the maintenance of a free market. To achieve this, they analyze the legal basis of the WTO's Antidumping Agreement in Peru, they explain the theories and effects of dumping practices and, at last, they critically analyze the antidumping measures taken by Indecopi against Chinese footwear. The authors close the article by recommending ways in which Indecopi can improve its performance in these types of matters.

KEYWORDS: Indecopi; dumping; World Trade Organization; free market.
En el Perú, la autoridad de competencia, el Indecopi, mantiene al mismo tiempo la función de velar por la libre competencia y la de proteger a los mercados de las supuestas distorsiones de las prácticas del dumping. Ello es incoherente ya que ambas disciplinas se inspiran en principios opuestos: una favorece el libre intercambio mientras que la otra tiende al proteccionismo.

En el presente artículo, los autores abordan las políticas de dumping del Indecopi y cómo estas pueden colisionar con el respeto a la libre competencia. Para ello, analizan la base legal del Acuerdo Antidumping de la OMC en el Perú, exponen el sustento teórico y los efectos de la práctica del dumping $y$, finalmente, desarrollan un análisis crítico de las medidas antidumping que el Indecopi ha impuesto sobre el calzado chino. Este marco permite a los autores elaborar una serie de recomendaciones para mejorar el funcionamiento del Indecopi en esta materia.

PALABRAS CLAVE: Indecopi; dumping; Organización Mundial del Comercio; libre competencia.

* Economista. Doctor en Economía Agraria por la Oklahoma State University. Ex Vicepresidente de la Comisión de Libre Competencia del Indecopi y ex Gerente General de Osiptel. Socio-Gerente del área de Consultoría Económica en APOYO Consultoría (Lima, Perú). Contacto: gcannock@apoyoconsultoria.com.

** Economista. Consultor del área de Consultoría Económica en APOYO Consultoriía (Lima, Perú). Contacto: rezcurra@ apoyoconsultoria.com

Nota del Editor: El presente artículo fue recibido por el Consejo Ejecutivo de THËMIS-Revista de Derecho el 1 de julio de 2020, y aceptado por el mismo el 2 de octubre de 2020 . 


\section{INTRODUCCIÓN Y MOTIVACIÓN}

El Instituto Nacional de Defensa de la Competencia y de la Protección de la Propiedad Intelectual (en adelante, Indecopi), como autoridad de protección de la libre competencia y de la propiedad intelectual tiene bajo su ámbito varias competencias funcionales como la represión de conductas anticompetitivas, la protección de derechos de propiedad intelectual, la protección al consumidor, la disminución de barreras burocráticas, la protección ante conductas de competencia desleal, la conducción del sistema concursal de las empresas, la corrección de distorsiones por prácticas de dumping y subvenciones.

Una apreciación general de dichas competencias permitiría concluir que están alineadas a la promoción del mercado y a corregir fallas de mercado como posiciones de dominio, información asimétrica, tratamiento de externalidades positivas como la innovación en lo propiedad intelectual, barreras a la entrada y de salida al mercado. Sin embargo, habría en nuestra opinión una excepción: la función relacionada a la eliminación de las supuestas distorsiones del dumping, la cual no es necesariamente consistente con la promoción o protección de la libre competencia.

Así, los objetivos de estos dos tipos de intervenciones son distintos, incluso opuestos. Mientras que el de dumping resuelve con la imposición de medidas antidumping dirigidas a corregir supuestas distorsiones que en última instancia constituyen una protección a los productores de la "rama de producción nacional" en perjuicio de las industrias demandantes o de los consumidores nacionales; por otro lado, las intervenciones de libre competencia están dirigidas a preservar el proceso competitivo y el bienestar de los consumidores.

Las metodologías de análisis para determinar si las conductas denunciadas han sido incurridas también difieren significativamente. En los casos de libre competencia se definen mercados relevantes de producto, geográficos, y de comercialización. Así, se analizan los efectos sustitución entre los productos para la determinación del mercado relevante, así como el grado de sustitución por el lado de la oferta para luego evaluar si el denunciado posee posición de dominio en dichos mercados relevantes, y finalmente si la práctica denunciada fue efectivamente incurrida y tuvo un efecto causal negativo sobre el proceso de la competencia en el mercado. La atribución del efecto causal generalmente se plantea y estima con la especificación de un escenario contrafactual, es decir, qué hubiera pasado en el mercado relevante si no hubiera ocurrido la práctica denunciada. Las em- presas infractoras de la libre competencia deben cesar dichas conductas y son sujetas a sanciones monetarias.

Por otro lado, en los casos de dumping -las que serían parte de la familia de prácticas de conductas predatorias- se basan en un procedimiento y una metodología acordadas en el Acuerdo de Antidumping de la Organización Mundial del Comercio (1994) (en adelante, Acuerdo Antidumping y OMC, respectivamente), en los cuales, para el caso de dumping, se requiere probar que el precio de exportación de la empresa exportadora denunciada está por debajo del precio vendido en el mercado doméstico de origen, y que ello ha originado un daño a la industria nacional. No se requiere demostrar que existe posición de dominio, ni tampoco si existe una motivación de afectar la competencia para excluir a competidores mediante el dumping o las subvenciones. La causalidad se muestra relacionando los cambios en las tendencias de los niveles de importación agregados, de los precios de importación, y de la participación en el mercado de importaciones en las empresas de origen extranjero denunciado con los cambios en la producción, ventas, y utilidades.

De hallarse que se ha incurrido en prácticas de dumping, se imponen derechos antidumping, que afectan a las importaciones de todo el mercado de las partidas arancelarias del país de origen por un plazo de hasta de cinco años. Estos pueden ser prorrogados si se mantienen las condiciones de dumping y de daño a la industria nacional supuestamente afectada.

Esta investigación sostiene que no es consistente que la autoridad de competencia nacional mantenga al mismo tiempo la disciplina de libre competencia con la de dumping sin realizar modificaciones a esta última. Ello es relevante en la medida que a la propia Comisión de Libre Competencia del Indecopi se le ha facultado en el 2015 mediante el Decreto Legislativo 1205, que recomiende a entidades del Estado medidas que reestablezcan o promuevan la libre competencia.

Esta eventual inconsistencia ha sido señalada también por Sykes y Cooper (1998) para el caso de los Estados Unidos de América, quienes realizaron un análisis histórico de la evolución de la legislación y de la política económica sobre libre competencia y antidumping. Concluyen que, si bien ambas tuvieron raíces comunes en su origen, han evolucionado de manera muy distinta, estando las decisiones de las autoridades en la primera basada en sólidos principios económicos mientras que la segunda ha girado hacia un objetivo pro- 
teccionista y basada menos en una lógica de eficiencia económica. Pierce (2000) refiere también a varias investigaciones sobre las inconsistencias de las políticas sobre el dumping con las de la libre competencia.

Desafortunadamente, la revisión que realizó la Organización para la Cooperación y el Desarrollo Económicos (en adelante, OCDE) en el 2018 sobre la política de competencia, si bien resalta la multiplicidad de funciones del Indecopi -aspecto criticado desde sus orígenes- no analiza la coherencia entre estas funciones. Recientemente el caso de la publicidad de octógonos ha sido un ejemplo de que funciones como la de eliminación de barreras burocráticas y la de protección al consumidor pueden colisionar. Pero este caso parece ser más de naturaleza anecdótica que un caso de colisión estructural como lo es el tratamiento del dumping y de la libre competencia.

Los casos sobre dumping y subvenciones son los que más han originado diferencias entre los países miembros de la OMC. Aproximadamente el 50\% de los casos que conoce el panel de solución de diferencias de la OMC trata de estos temas. Los procesos de liberalización que implicaron una reducción en los aranceles y los tratados bilaterales de libre comercio también coincidieron con un mayor empleo de medidas antidumping en los países ${ }^{1}$.

\section{EL ENTORNO DEL ACUERDO ANTIDUMPING DE LA OMC Y SU BASE LEGAL EN EL PERÚ}

\section{A. Antecedentes}

La aplicación de la evaluación de las prácticas de dumping y subvenciones se origina en el Acuerdo General de Tarifas y Aranceles (en adelante, GATT por su acrónimo en inglés). El GATT constituyó un conjunto de acuerdos relacionados al comercio internacional que fueron realizados en varias largas rondas de negociación luego de la segunda guerra mundial entre 1947 y 1994 para minimizar las reacciones proteccionistas de los países.

En la ronda de negociaciones finales del GATT se acordó también la creación del Organismo Mundial del Comercio para que básicamente aplicara dicho acuerdo y condujera nuevas negociaciones para promover el comercio de bienes y servicios bajo un enfoque de acuerdos multilaterales entre los países miembro.

En el título VI del GATT de 1994 se desarrolla el acuerdo sobre dumping. Para determinar que es necesario imponer un derecho antidumping, se debe estimar el margen del dumping, y el daño a la Rama de Producción Nacional (en adelante, RPN). Respecto al dumping, el acuerdo tiene un detalle sobre su definición y las maneras de estimar el denominado margen de dumping según la información disponible. Así, el dumping es la diferencia del precio de exportación a un país miembro menos un valor considerado como "normal" de un precio de un producto similar destinado al mercado interno del país exportador (Acuerdo general sobre aranceles aduaneros [GATT], 1994, artículo VI). Si no se vende el producto en el mercado interno del país exportador o dicho mercado es muy pequeño, el acuerdo permite emplear los precios de exportación a terceros países, el precio de la primera reventa del producto en terceros países, o a la estimación del costo de producción promedio de las empresas exportadoras que consideren costos variables y costos fijos, incluyendo los gastos administrativos e indirectos, y utilidades; siempre que se apliquen principios de contabilidad generalmente aceptados. La comparación del precio de exportación con el valor normal se debe hacer en el mismo nivel comercial (por ejemplo, ex fábrica).

Respecto a la determinación del daño a la RPN, el criterio empleado se desdobla en mostrar el volumen de importaciones bajo el supuesto dumping, y su efecto sobre los precios del producto vendido por la RPN. Sobre las importaciones, el indicador se basa en el volumen importado, ya sea en términos absolutos o con relación a la producción o consumo del país importador; y con respecto al precio, se analiza si el precio de las importaciones con el supuesto dumping se encuentra por debajo al de los productores nacionales, o si tuviese el efecto de disminuirlos o que impida que suban los precios. La repercusión del dumping sobre el desempeño de la RPN se puede reportar a través de un conjunto de indicadores como ventas, producción, utilidades, participación de mercado, uso de la capacidad instalada, empleo, salarios, los flujos de caja, entre otros.

Es relevante notar que el acuerdo explícitamente menciona que se debe examinar otras razones distintas al dumping que hayan podido impactar sobre el desempeño de la RPN, como por ejemplo los precios y volúmenes no sujetos a dumping, los cambios en la demanda, la tecnología, y la productividad de la RPN.

Otros puntos del acuerdo que son relevantes destacar son los siguientes:

Véase Voon (2007) 
i) el proceso de investigación se inicia a solicitud de la RPN, quienes deben acreditar que representan al menos el $25 \%$ de la RPN respectiva;

ii) es posible establecer medidas provisionales como derechos o una garantía que no exceda el margen de dumping; $y$,

iii) el acuerdo contempla también un proceso por cambios de circunstancias en el caso que se identifique una situación de un daño importante e inminente. Para ello, la autoridad competente debe revisar si existe un fuerte aumento de las importaciones sujetas a dumping; una alta capacidad de exportación disponible de las empresas del país exportador; y que las importaciones tengan como efecto bajar los precios internos y provoque a su vez mayores importaciones (GATT, 1994).

Con respecto al marco normativo peruano, es interesante notar que desde el año 1991, previo al GATT de 1994, se aprobó el Decreto Supremo 133-91-EF en el cual se introdujeron la evaluación de dumping y la determinación de los derechos antidumping en el país. En los considerandos de la norma, se justificó la necesidad de la norma sobre la base de que la Constitución Política del Perú de 1979 -vigente en ese momento- estableció que en una economía social de mercado el Estado no puede permitir actos que distorsionan la competencia como el dumping y las subvenciones. Esta norma definió el dumping y la manera de estimarlo de manera similar a la que se aprobó luego en el GATT de 1994; y creó la Comisión de Dumping y Subsidios (en adelante, CDS). La CDS dependía del Ministerio de Economía y Finanzas. La norma otorgó la discreción a la CDS de determinar en última instancia el margen de dumping en el caso de que no fuesen aplicables los diversos métodos para calcular el valor normal y el margen de dumping.

En diciembre de 1994, mediante la Resolución Legislativa 26407, el Congreso Constituyente aprobó el acuerdo para crear la OMC, y los acuerdos comerciales multilaterales del GATT de 1994 aprobados en el acta de negociación de Uruguay, que incluyen el acuerdo sobre dumping. El acuerdo fue reglamentado y modificado sucesivamente por el Decreto Supremo 043-97EF, el Decreto Supremo 144-2000-EF, y el Decreto Supremo 225-2001-EF.
En 1992, se creó el Indecopi. Si bien en sus funciones se le otorgó el mandato de proteger a los mercados de prácticas que generen competencia desleal y de aquellas otras que afecten a consumidores y a otros agentes del mercado, no se especificó expresamente el deber de proteger a los mercados de distorsiones de las prácticas de dumping y subsidios. Sin embargo, su norma de creación estableció como una de sus áreas funcionales la Comisión de Fiscalización de Dumping y Subsidios. A dicha comisión se le encargó la aplicación del Decreto Supremo 133-91-EF y sus modificatorias ${ }^{2}$.

El aún vigente Decreto Supremo 006-2003-PCM (2003) ha ordenado en una sola norma la reglamentación nacional sobre el dumping. Sobre los aspectos a resaltar de dicha norma, se tiene que reiterar que los dumping y subsidios pueden ser prácticas anticompetitivas según lo establecido por la Constitución Política del Perú ${ }^{3}$. Introduce el concepto de situación especial de mercado, que ocurre cuando la autoridad nacional considera que los costos, precios, y las condiciones de mercado en el país de origen, es decir el país exportador, están distorsionados. Establece que la investigación sobre la existencia de dumping puede ser de oficio si los productores no están organizados o por razón de interés nacional. También, recoge la opción de que las empresas exportadoras ofrezcan un compromiso sobre el precio de exportación en un nivel tal que evite la imposición de un derecho antidumping por la autoridad nacional. La norma precisa que los derechos antidumping tienen condición de multa. Asimismo, dispone que la vigencia de los derechos de antidumping no puede ser mayor a cinco años salvo que se siga previamente un procedimiento de sunset review o de examen de expiración, en el cual se analiza si es probable que el dumping continúe luego de la expiración, así como si es probable que el daño a la industria nacional persista de eliminarse los derechos antidumping (Decreto Supremo 006-2003PCM, 2003).

Debido a que un alto número de casos investigados de dumping en el mundo han involucrado a las exportaciones de la República Popular China (en adelante, RPC), de los cuales detallamos uno más adelante, es conveniente resumir el marco aplicable para ese país. Antes del 2001, año en el cual la RPC accedió a la OMC, se trataba a este país como una economía que no era de mercado. En ese sentido, el Estado peruano definió varios cri-

\footnotetext{
La precisión se hizo recién en 1996 mediante el Decreto Legislativo 807.

3 Regía la nueva Constitución Política aprobada en 1993.
} 
terios de evaluación, como por ejemplo, si los precios se formaban en libre competencia con base en la oferta y demanda, si había interferencia del Estado en la determinación de costos y precios, la existencia de normas de acceso y salida del mercado, entre otros ${ }^{4}$. La implicancia para estos fines fue que la autoridad nacional podía determinar el valor normal no sobre la base de la información de los productores de la RPC, sino de un tercer país, no necesariamente es comparable ${ }^{5}$, a efectos de estimar el margen de dumping.

Con el acuerdo de adhesión a la OMC (2001), la RPC se comprometió, entre otros puntos, a:

i) no establecer precios diferenciados según el destino de venta de los productos, sea al mercado interno o al de exportación;

ii) no emplear controles de precios para proteger a los productores nacionales, y que los precios de bienes transables se determinen por las fuerzas del mercado;

iii) que en las zonas económicas especiales se cobraría los mismos derechos y políticas para bienes importados que se apliquen en el resto del país;

iv) que las empresas estatales chinas no intervengan de manera tal que afecten la cantidad, valor u origen del comercio;

v) notificar de cualquier subsidio que establezca definido según el acuerdo de subvenciones de la OMC; $y$,

vi) se acordó un mecanismo de salvaguardia de transición que los países importadores miembros podrían invocar que regiría doce años desde la fecha de acceso para ciertos productos de exportación de la RPC (Organización Mundial del Comercio, 2001).

Por otro lado, las medidas de los países importadores contra la RPC que sean inconsistentes con los acuerdos de la OMC, deberían ser dejadas sin efecto. Finalmente, con respecto a la determinación del valor normal para el cálculo de dumping se acordó que si los productores chinos demuestran que las condiciones de mercado prevalecen en la industria del producto similar bajo investigación, la autoridad del país importador emplearía los pre- cios y costos internos de la RPC; de lo contrario la autoridad del país importador puede emplear otra metodología para comparar los precios.

\section{SUSTENTO TEÓRICO Y EFECTOS DE LA PRÁC- TICA DEL DUMPING}

\section{A. Monopolio en país exportador}

Una primera cuestión para evaluar sobre el dumping es si es en realidad una práctica anticompetitiva. La teoría microeconómica básica ${ }^{6}$ brinda el fundamento más común y estándar para explicarlo. Lo que se conoce como dumping constituye un caso de discriminación de precios de una empresa que es monopolista en su mercado local, la cual, aprovechando que los mercados de destino están separados, que los costos son los mismos y que no hay arbitraje, le permite cobrar un precio monopólico en su mercado doméstico y un precio menor en el mercado de exportación dependiendo de las diferencias en las elasticidades de la demanda.

En ese sentido, el monopolista prefiere restringir la venta en su mercado doméstico y elevar el precio interno por encima de su costo marginal; mientras que vende en el mercado de exportación una mayor cantidad, pero a un menor precio. Asumiendo razonablemente que el mercado global es competitivo, es relevante notar que el precio de exportación de la empresa es igual al precio internacional, lo que es el costo de oportunidad tanto para el exportador como para el importador en el país de destino.

En ese sentido, el precio para el país importador no es mayor que el internacional. Por lo tanto, no se genera un daño a los importadores, y por extensión tampoco a los productores nacionales por la supuesta práctica del dumping. El escenario contrafactual, es decir, sin dumping, implicaría que el precio doméstico en el país exportador bajaría al mismo nivel que el precio internacional, pero el precio de exportación en el mercado de destino sería el mismo respecto a la situación con dumping. Por tanto, si el dumping se considera como una práctica de competencia desleal, ello se debería a que la participación de la producción nacional en el país importador disminuye. No obstante, aquello no es una práctica anticompetitiva; dado que el dumping no excluye a los productores de la RPN vía una práctica de precios

\footnotetext{
Véase el Decreto Supremo 225-2001-E.
}

5 Mai (2002) identifica varios casos donde los terceros países no son comparables y que han tendido a elevar la estimación del margen de dumping.

6 Para mayor información véase a Kaf. 
predatorios. El precio internacional tendría que estar por debajo del costo marginal del producto o de algún indicador similar, lo que es sumamente improbable debido a que cualquier exportación sería insostenible.

En su defecto, para que ocurra un impacto sobre el mercado doméstico del país importador, el país exportador tendría que expandir su oferta exportable en una magnitud tal que influya a generar una reducción en los precios internacionales. Esta expansión de la participación de mercado de las empresas exportadoras sería consistente con la libre competencia si se logra aprovechando mayores economías de escala o adopción de innovaciones que les permita alcanzar un menor costo marginal.

\section{B. Dumping estratégico y dumping predatorio}

Willig (1998) profundiza en el conjunto de las posibles razones que podrían dar lugar al dumping. Su mensaje central es que algunas de ellas podrían ser coherentes con la libre competencia, mientras que otras no. Amplía primero el análisis estándar al incluir no solo al monopolio en el país exportador, sino también al evaluar si dicho resultado puede darse bajo colusión o de competencia oligopólica. Además, propone una tipología de dumping de cinco categorías según su motivación:

i) la expansiva, mediante la cual los exportadores pretenden crecer en mercados de exportación cobrando por debajo de los costos de transporte;

ii) la cíclica, en la cual por razones estacionales o cíclicas los exportadores enfrentan un exceso de capacidad instalada y la emplean para elevar su producción y cubrir sus costos fijos;

iii) la del Estado, que corresponde a empresas estatales que exportan a menor precio con el fin de acceder a divisas y con ello levantar la restricción que pudieran tener con la tasa de cambio;

iv) la estratégica, mediante la cual el mercado interno del país exportador está cerrado a terceros países; y que, además, la industria del país exportador se caracteriza por contar con importantes economías de escala, de aprendizaje e innovación con respecto a su tamaño de mercado -ello permitiría que los exportadores puedan penetrar en los mercados de importación a menores precios, y que los productores de los países importadores no tengan la posibilidad de alcanzar las eco- nomías de escala por no tener acceso al mercado del país exportador-; $y$,

v) la predatoria, mediante la cual las empresas exportadoras pretenden excluir irreversiblemente a los productores del país importador (Willig, 1998).

Para Willig, los tres primeros tipos de dumping no se basan en una monopolización o exclusión del mercado y son consistentes con la libre competencia, dado que en el país importador habrá competencia generada por las opciones en el mercado internacional y por los productores nacionales (1998). Estos dumpings, a pesar de que reducen la renta de los productores, si se comportan como lo harían en un mercado en competencia, producen una pérdida menor a la ganancia que obtienen los consumidores de adquirir los bienes a menores precios.

El dumping estratégico en cambio puede ser anticompetitivo debido a que:

i) los productores del país importador no pueden acceder al mercado del país exportador;

ii) producen en su mercado con mayores costos;

iii) no pueden competir en iguales condiciones con las empresas exportadoras en su mercado interno; $y$,

iv) los consumidores del país importador pueden quedar sujetos al poder de mercado de las empresas del país exportador si las empresas nacionales no pueden competir efectivamente.

Con respecto al dumping predatorio, Willig recuerda las condiciones estructurales que tendrían que existir en el mercado del país importador para que este tipo de dumping sea efectivo:

i) que el mercado esté concentrado para que la empresa predadora pueda tener poder de mercado una vez excluidos a los productores nacionales;

ii) que existan barreras a la entrada en la producción nacional;

iii) que existan barreras para la reentrada de los productores que tuvieron que salir por el dumping; $\mathrm{y}$,

iv) el dumping debe estar acompañado por otras estrategias de exclusión dado que solo 
usando la variable precio no necesariamente se excluye en el largo plazo a los productores

\section{Comportamiento mercantilista}

Uno de los potenciales efectos indeseados de las aplicaciones de antidumping es la oportunidad que genera para comportamientos mercantilistas. Es decir, en lugar de que la motivación del productor sea competir en el mercado, estos buscan rentas brindadas por los gobiernos. Este comportamiento fue identificado y teorizado inicialmente por Tullock (1967) y Krueger (1974), los cuales toman temas de comercio internacional como ejemplo.

Para el caso específico de dumping, Anderson (1994) demuestra el caso del 'lobby en dominó'. En este, los lobbies tanto en el país importador como en el exportador actúan estratégicamente a través de sus gobiernos para generar normas $y$ para hacerlas cumplir a través del antidumping y las restricciones voluntarias a la exportación, siendo esta última figura planteada como una política de sustitución al derecho antidumping.

Evidentemente, la posibilidad de que se establezca un derecho antidumping que eleve el costo de importación a la competencia de los productores nacionales constituye un fuerte incentivo para realizar esfuerzos con la intención de que ello efectivamente ocurra. Estos esfuerzos pueden llegar a ser equivalentes a la renta esperada (o evitada según el punto de vista) de la imposición del derecho antidumping.

El reciente sustento que realizó el mismo Indecopi ${ }^{7}$ para modificar el capítulo económico de la Constitución, con el fin de mejorar su autonomía, sugiere que el mercantilismo es un riesgo evidente. Así, cita a un estudio que reporta la falta de autonomía del Indecopi en dos casos sobre dumping y subsidios, uno que involucró a un gremio empresarial que pudo obtener una medida proteccionista, y otro involucró a una entidad del Poder Ejecutivo que buscó reponer la reducción arancelaria que se realizó en el programa de liberación comercial con la imposición de derechos compensatorios (Indecopi, 2020).

\section{Acuerdos colusorios y dumping}

Vinculado al punto anterior, otra incoherencia de la política sobre dumping es que tiende a elevar el riesgo de acuerdos colusorios. Pierce (2000) analiza cómo la política de dumping puede ser empleada para crear una colusión en un mercado. Refiere el caso del ferro-silicón en los Estados Unidos, en el cual los productores nacionales ante la presencia de competencia vía importaciones de cinco países acordaron formar un cartel entre ellos y elevaron los precios.

Cuando subieron las importaciones a menores precios que el precio acordado, el cártel interpuso una demanda de dumping ante la agencia federal competente. La agencia estableció derechos antidumping que tuvo el efecto de excluir a las importaciones de esos países. Más adelante, cuando Brasil inició sus exportaciones, el cartel invitó a los exportadores del Brasil que se unieran, o en caso contrario iniciarían una denuncia por dumping. Los exportadores brasileños se negaron, y el cartel hizo efectiva su denuncia, y las autoridades le dieron la razón al cartel, imponiendo derechos antidumping a las empresas brasileñas. Finalmente, posteriormente las autoridades de competencia y las judiciales identificaron el acuerdo e iniciaron acciones administrativas y penales contra el cartel.

Pierce sostiene que las normas sobre dumping tienden a favorecer la formación de carteles debido a varias razones:

i) las acciones son interpuestas contra productos que son homogéneos -es más fácil formar carteles en productos homogéneos, y las investigaciones de dumping definen los productos similares de manera muy específica según partida arancelarias-;

ii) al excluir a las empresas importadoras del mercado relevante, reducen el número de empresas, lo cual tiende o también a facilitar la formación del cartel;

iii) los gobiernos se inclinan a proteger a las empresas locales;

iv) la sola amenaza de que se impongan derechos antidumping puede excluir a nuevos competidores que no son parte del cartel; $y$,

v) estas amenazas de dumping pueden constituirse en instrumentos de monitoreo y supervisión del cartel (2000).

De la revisión del acuerdo de la OMC y del marco legal, es posible identificar algunos puntos específicos que podrían implicar ciertos riesgos adicionales:

i) que sean los productores organizados y formalmente legales de la RPN los que puedan

Véase la Resolución de Presidencia 038-2020-INDECOPI/COD y Documento de Trabajo Institucional 003-2020. 
presentar una solicitud de investigación de dumping;

ii) que estos puedan compartir información sobre el desempeño de su mercado para poder sustentar los supuestos daños a su industria;

iii) debido al hecho que algunos procedimientos como el sunset review se deben iniciar con cierta anticipación, lo cual implica un monitoreo del mercado por parte de los denunciantes;

iv) que las empresas del país exportador pueden presentar una oferta de precios o de restricción voluntaria de exportaciones para que sea eliminado el derecho antidumping; $y$,

v) que puedan tener un acuerdo de precio o de distribución en el mercado interno una vez establecido el derecho antidumping a la competencia.

\section{E. El valor normal y libre competencia}

El concepto de valor normal empleado en las investigaciones de dumping para calcular el precio interno del país exportador denunciado -en muchos casos no existe disponibilidad de información de precios del producto denunciado en el mercado doméstico- resulta extraño en el análisis de mercados y de la competencia si se le interpreta como un valor intrínseco. En un mercado competitivo, implicaría que no se altera ningún factor que influye sobre la oferta o la demanda de un mercado. Es decir, por un lado, que se mantienen inalterables el comportamiento y preferencias de los consumidores al igual que sus niveles de ingresos; $y$, por el otro, que la estructura de la oferta se mantiene con los mismos factores de producción, número de empresas y sin cambio técnico. Cualquier cambio sobre alguna de esas variables influiría sobre el valor normal. También podría ocurrir un valor inalterable en algunas situaciones muy extremas cuando el precio es completamente determinado, ya sea por la oferta o la demanda. Ello sucedería en un mercado con muchos bienes sustitutos o con una industria con costos marginales y economías de escala constantes, situaciones que difícilmente ocurren en casos reales.

Por otra parte, si el valor 'normal' se volviese un valor inmutable, este perdería su rol como señal de información para que los agentes participantes en el mercado tomen decisiones, lo cual es central para los mercados como mecanismos de coordinación eficientes.

Otra interpretación es que se considere un valor normal aquel que resulte de la libre concurrencia e interacción de los agentes del mercado. No obstante, ello nos daría como resultado simplemente el precio como equilibrio determinado en el mercado relevante. Habría tantos precios y valores normales como mercados relevantes de cada producto. No tendría sentido calificar a alguno como normal salvo que sea una decisión arbitraria.

Por ello, las metodologías sugeridas en la normativa del dumping son esencialmente arbitrarias y no están alineadas a los conceptos empleados en libre competencia. Postular que el valor normal de un producto es el costo de producción, incluyendo costos fijos, administrativos e indirectos, más un estimado de utilidad para determinar un comparador de precio, ignora que los precios en competencia se fijan según sus costos marginales, incluyendo los costos de oportunidad. Además, la valoración no está únicamente en función de los costos, sino de la disposición a pagar de los consumidores. En la medida en que los costos marginales no son directamente observables, y que son cambiantes según las condiciones del mercado, es un ejercicio fútil tratar de estimarlos para contar con estimados del valor normal.

Por otro lado, no es posible identificar si los cambios en los precios de exportación se deben a una consecuencia de ventajas comparativas estáticas y dinámicas del país exportador o de un supuesto dumping. La estimación de valores normales y de sus correspondientes márgenes de dumping y eventualmente de derechos antidumping, están sujetos a altos márgenes de error.

\section{EL CASO DE DUMPING DE CALZADO DE LA RPC}

En esta sección presentaremos los antecedentes del caso, y luego un análisis crítico focalizado en tres temas.

\section{A. Antecedentes, procesos, y resoluciones}

En el siguiente cuadro se muestra las principales resoluciones de la CDS y de la Sala de Competencia relacionadas con el caso de dumping de calzado de la RPC.

Lo más resaltante del caso, sin duda, es que se evidencia más de veinte años con derechos antidumping impuestos a calzado de chalas y sandalias de la RPC, a pesar de que el acuerdo de la OMC estipula que, en principio, no deben mantenerse por más de 5 años. Si bien ha habido algunas variaciones en las subpartidas arancelarias -excluyéndose a las de material superior de cuero y a las originarias de Taiwán- esencialmente, por su naturaleza, ha sido el mismo caso. 
Cuadro 1

Resoluciones sobre el caso de dumping en calzado de la RPC 1997-2019

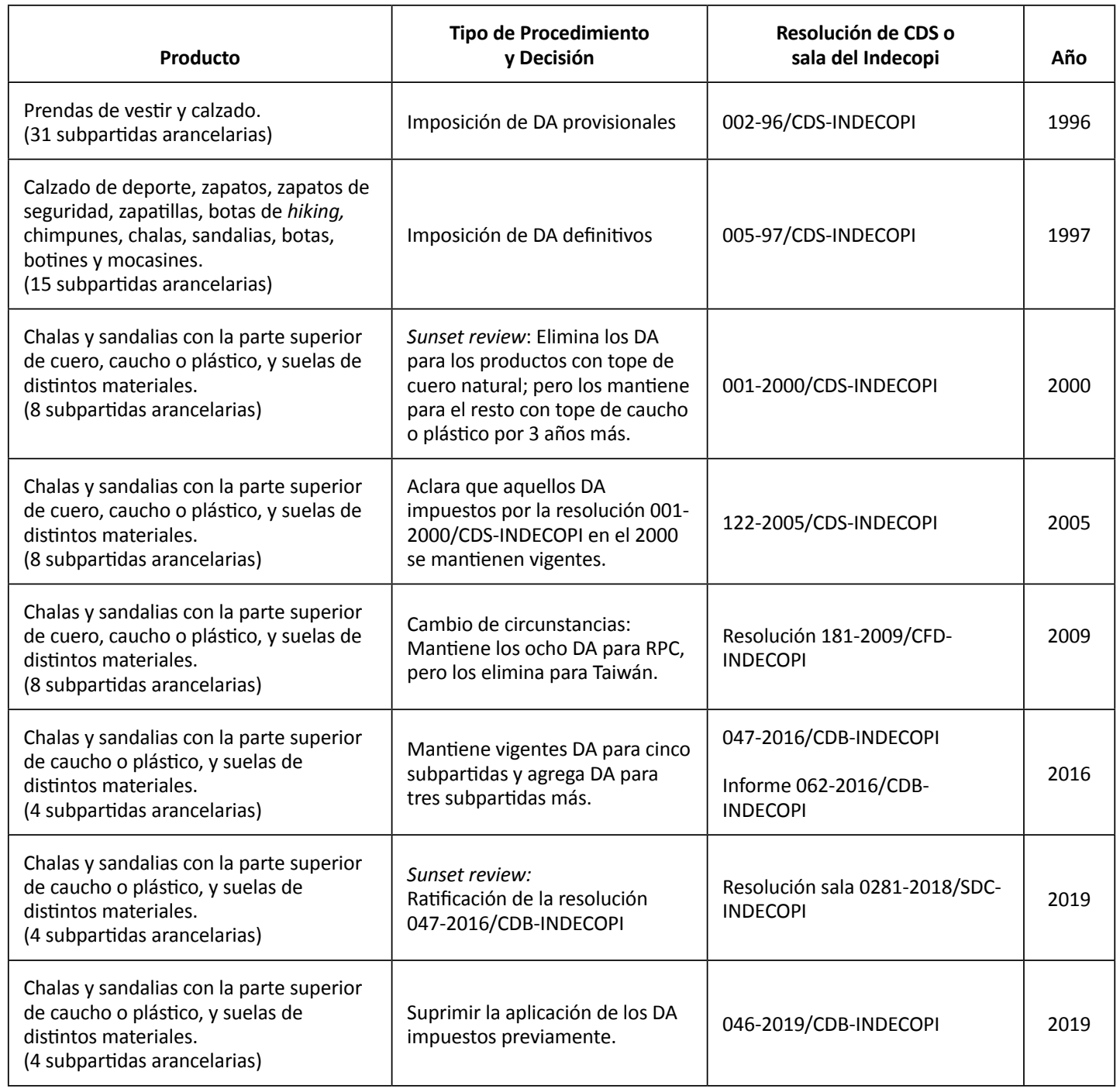

Fuente: Elaboración propia a partir de la información del Indecopi.

En adición, el caso muestra que se han aplicado el conjunto de procedimientos contemplados en el acuerdo de la OMC: imposición de derechos provisionales, el establecimiento de derechos definitivos, procedimiento por cambios de circunstancias, y por expiración de medidas. Ello contribuyó a extender la vigencia de los derechos antidumping.

En 1996, la CDS del Indecopi inició una investigación de oficio para analizar partidas arancelarias de prendas de vestir y calzados provenientes de la RPC que podían estar incurriendo en prácticas de dumping (Resolución 001-96-INDECOPI/CDS). La investigación de oficio se justificó aduciendo tres premisas:

i) que el volumen de prendas y calzados provenientes de la RPC había ido en aumento; ii) que la economía de la RPC mostraba una serie de distorsiones que impedían considerarla una economía de libre mercado; $y$,

iii) que al comparar los precios Free on Board (en adelante, FOB) de exportación al Perú de diversas prendas y calzado con el precio de referencia calculado en base a los precios de importación de otros países de la región, se observó que los primeros eran menores y que, por lo tanto, constituían un indicio de la existencia de dumping (Resolución 001-96-INDECOPI/CDS, 1996).

Así, como medida inicial, resolvió que 31 subpartidas arancelarias (en adelante, SPA) fueran sujetas a derechos antidumping provisionales. 
El siguiente año, el Indecopi dio por concluida la investigación y declaró que quince de estas subpartidas, que incluían distintos tipos de calzado proveniente de la RPC, debían de estar sujetas al pago de derechos antidumping definitivos ${ }^{8}$. La metodología empleada para calcular el valor a pagar del derecho antidumping fue el cálculo de márgenes de dumping. Estos márgenes se obtuvieron como resultado de la división de la diferencia entre el precio normal y el valor de importación, entre el valor de importación. El valor normal de los productos analizados fue calculado sobre la base de los resultados de un trabajo realizado por el Centro de Investigación de la Universidad del Pacífico; mientras que el precio de importación se obtuvo de las bases de datos provistas por Aduanas. No obstante, debido a la alta dispersión de los precios FOB encontrado en las distintas partidas arancelarias, los derechos antidumping fueron fijados en base a rangos que utilizaban como criterio el valor de los márgenes de dumping calculados.

En el 2000, la Corporación del Cuero, Calzado y Afines, -en representación de la asociación de pequeños y medianos fabricantes de calzado- solicitó a la CDS iniciar una investigación por prácticas de dumping en las importaciones de productos de esta categoría por parte de la RPC, Taiwán y Hong Kong (Resolución 001-2000/CDS-INDECOPI). Asimismo, la petición también contemplaba la revisión del examen de los derechos antidumping vigentes y su cuantía?

La Secretaría Técnica concluyó que existía dumping en los productos importados por Taiwán y que los derechos antidumping vigentes para las importaciones chinas no contemplaban la diferencia real entre el precio del calzado en el país originario y el precio en el país importador. Además, la participación en el total de importaciones tanto de la RPC como de Taiwán, se incrementó considerablemente desde 1997, mientras que la producción de la RPN de calzado disminuyó en $19.91 \%$. Como consecuencia, la CDS resolvió la aplicación de derechos antidumping para aquellos productos de calzado provenientes de Taiwán y la modificación al alza del monto recaudado en los derechos antidumping del calzado proveniente de la RPC (Resolución 001-2000/CDS-INDECOPI, 2000).

La Resolución 122-2005/CDS-INDECOPI del 2005 resolvió que los derechos antidumping impuestos en la última revisión se mantengan para el caso de
Taiwán y la RPC. Esta resolución tuvo un carácter aclaratorio más que un análisis técnico, dado que tuvo como objetivo ratificar la continuidad de las conclusiones del 2000. El sustento detrás de esta acción se basó en que los productos seguían presentando características de dumping y, por lo tanto, afectando la RPN de esta industria. Cuatro años después la Resolución 181-2009/CFD-INDECOPI resolvió la solicitud presentada por la Asociación Nacional de Pequeños Importadores de Sandalias y Chalas (en adelante, ANPISCH) sobre un examen de cambio de circunstancias con el fin de mantener o modificar los derechos acordados previamente.

En lugar de investigar la existencia de la práctica, el daño sobre la RPN y la relación causal existente entre las importaciones y la producción nacional; en este tipo de examen, el Indecopi debe determinar si es necesario o no mantener las medidas implementadas. Con respecto a Taiwán, la CDS concluyó que resultaba improbable que el dumping y el daño a la RPN vuelvan a aparecer en caso se eliminen los derechos antidumping relacionados (Resolución 181-2009/CFD-INDECOPI, 2009). Por otro lado, una decisión diferente fue tomada con respecto a la RPC. En este segundo caso, la CDS resolvió por seguir aplicando derechos antidumping debido a que concluyeron en su análisis prospectivo que la RPC seguía teniendo incentivos para practicar y seguir practicando en un futuro el dumping (Resolución 181-2009/ CFD-INDECOPI, 2009).

El procedimiento del 2009 estableció que los derechos antidumping impuestos a productos de la RPC tengan una vigencia máxima de cinco años, tras los cuales debían de ser sujetos a revisión. De esta forma, la siguiente investigación sobre las características de mercado del caso se dio en el 2014, como parte del sunset review solicitado por representantes de la RPN. No obstante, la resolución del Indecopi no tomaría una decisión hasta el 2016. La Resolución 047-2016/CDB-INDECOPI se centró en la capacidad que podían tener los productos provenientes de la RPC de dañar la RPN y cómo, efectivamente, los precios estaban distorsionando el mercado peruano. Bajo argumentos similares a los usados previamente, la CDS concluyó que los productos considerados bajo la categoría de chalas y sandalias con la parte superior de caucho o plástico seguirían estando sujetos a los derechos antidumping, eliminándose para chalas y sandalias con la parte superior de cuero. Asimismo, la CDS tomó la decisión de fijar la fecha de re-

\footnotetext{
Los derechos antidumping provisionales para todas las SPA fueron suprimidos.

9 Cabe resaltar que Taiwán y China todavía no formaban parte de la OMC en este momento por lo que el plazo máximo de cinco años de vigencia para los derechos antidumping no aplicaba para ninguno.
} 
visión de los derechos cada tres años (Resolución 047-2016/CDB-INDECOPI, 2016).

Finalmente, en el 2019 se dio la última revisión del caso de calzado en dumping. Así, el sunset review del 2016 fue revisado por la CDS, la cual resolvió que las condiciones en las cuales se encontraba la RPN y las acciones por parte de los importadores chinos mantenían la supuesta causalidad sobre los efectos en la producción nacional de calzado. Luego del análisis, la Sala Especializada en Defensa de la Competencia mediante Resolución 0282-2017/ SDC-INDECOPI decidió ratificar los alcances de la resolución del 2016, manteniendo de esta forma los derechos antidumping para las sandalias y chalas importadas de la RPC vigentes.

No obstante, pocos meses después, la vigencia de estos derechos antidumping llegaría a su fin. Como se describió, los derechos antidumping tienen un plazo máximo de vigencia tras el cual, si es que no se concluye en una investigación que existe probabilidad de continuidad del dumping, estos deben cesar de existir. De esta manera, si bien hubo solicitudes por parte de empresas pertenecientes a la RPN para abrir un nuevo procedimiento de sunset review, la CDS decidió no considerarlos debido a temas de fondo y forma en los pedidos. Por parte de la forma, los requisitos exigidos por el Indecopi para abrir un procedimiento de esta naturaleza no fueron cumplidos por las empresas solicitantes; mientras que, por el lado de fondo, la entidad concluyó que la información económica disponible no era suficiente para evaluar la posible continuidad de estas prácticas.

Finalmente, es relevante mencionar que las empresas exportadoras de la RPC en diversos otros rubros como el textil y neumáticos han sido investigadas y se les han impuesto derechos antidumping en los últimos veinte años. Alrededor del $50 \%$ de los procedimientos de dumping en el país han involucrado a la RPC.

\section{B. Análisis crítico}

Los informes que sustentan las resoluciones contienen varios puntos que podrían ser comentados críticamente. Sin embargo, el análisis a continuación se centrará en la aplicación de algunos puntos señalados en las secciones anteriores: el margen de dumping, la informalidad en la RPN, y los impactos de la Zona Franca de Tacna (en adelante, Zofratacna).

\section{El margen de dumping}

Dentro del análisis realizado para determinar la existencia de dumping e imponer un derecho an- tidumping a una partida arancelaria, calcular el margen de dumping es un requisito indispensable. Este se puede entender como la diferencia entre el valor normal de un producto y el precio de importación de este, dividida entre el precio de importación mencionado. De esta forma, si el valor normal es mayor que el precio de importación registrado, estaríamos frente a un margen de dumping positivo que implica una práctica de dumping.

De acuerdo con los lineamientos de la OMC, el derecho antidumping no deberá ser mayor al margen de dumping generado por la práctica (GATT, 1994). Esto debido a que, de lo contrario, se estaría generando una barrera al comercio, lo cual iría en contra de lo que la entidad internacional busca promover. De este modo, se vuelve relevante repasar cuáles metodologías ha utilizado la autoridad para calcular el margen antidumping en las últimas dos décadas que tiene vigente el caso.

La primera metodología utilizada para analizar el caso de dumping de calzado fue contemplada en 1997. En el Informe 005-97-INDECOPI/CDS, para calcular el margen de dumping, se calculó el precio de importación y el valor normal de los productos. El precio de importación de los productos fue obtenido de la información de Aduanas y otros actores en el mercado. Luego, debido al estatus de la RPC como economía que no era de mercado para la OMC, se utilizó como base razonable para calcular el valor normal del producto un informe del Centro de Investigación de la Universidad del Pacífico (1997) y también, información referente a diversas cotizaciones de precios de dichos productos en Italia, Brasil, India y Corea del Sur. Sobre la base de estos datos, la CDS consideró que existía una diferencia considerable entre el valor normal y el precio de importación para once partidas arancelarias correspondientes a calzado proveniente de la RPC, determinando así la existencia de un margen de dumping. Asimismo, luego de investigar la existencia de un daño a la RPN, el Indecopi decidió imponer derechos antidumping a las partidas arancelarias mencionadas. No obstante, debido a la alta dispersión de los precios FOB reportados, los derechos antidumping definitivos impuestos fueron calculados en base a rangos de estos derechos.

La siguiente revisión de derechos antidumping en la que se consideró calcular los márgenes de dumping nuevamente ocurrió en el 2009, durante el procedimiento de cambio de circunstancias solicitado por ANPISCH. Este proceso requirió que la autoridad revisará si estos derechos debieran de seguir siendo aplicados. Si bien estos derechos fueron actualizados mediante la apli- 
cación de la regla del menor derecho o lesser duty rule ${ }^{10}$, el margen de dumping no fue calculado. Esto debido a que la CDS determinó que no existía información en el mercado para poder realizar este cálculo tras solicitar información a las empresas productoras chinas y no recibir respuesta de ellas. Asimismo, la CDS resaltó que, si bien calcular los márgenes de dumping podría contribuir al análisis, el Acuerdo Antidumping no establece que sea necesario calcularlo (1994). Esta misma línea argumentativa fue utilizada en la siguiente revisión en 2016, en el que la CDS optó nuevamente por obviar el cálculo del margen de dumping basándose en que no existía información suficiente.

De esta forma, la discusión para poder determinar la prevalencia del supuesto dumping se basó en la evolución de otros indicadores que la CDS consideró suficientes como carga probatoria. En este contexto, es importante cuestionar dos puntos: (i) si el cálculo de los márgenes de dumping es prescindible en estos casos o no y; (ii) si en realidad era inviable el cálculo de dichos márgenes en la última actualización del caso. La necesidad de calcular los márgenes de dumping nace de la directa relación existente entre el derecho antidumping y estos márgenes. Como se explicó, el derecho antidumping deberá ser igual al margen de dumping que está generando la empresa exportadora. Esto debido a que, de lo contrario, podría generarse una barrera al comercio. El problema nace de una revisión de derechos antidumping que no contempla el cálculo de estos márgenes. Como consecuencia, se desprende que una autoridad debería hacer lo posible por calcular estos márgenes.

Por otro lado, con respecto a la viabilidad del cálculo de estos márgenes, sí era posible calcularlos en base a información pública y empleando las pautas metodológicas del acuerdo de la OMC. Así, el precio de exportación de la RPC al Perú se puede recabar de información de aduanas, mientras que el valor normal se podía calcular empleando los precios de exportación o de venta de otros países tomados como países de referencia, o de los precios de importación de terceros países. Ambas metodologías son similares a la utilizada por la CDS en 1997, que es el cálculo del margen de dumping como el resultado de la división de la diferencia del valor normal y el precio de importación, entre dicho precio de importación. Cabe resaltar que ambas prácticas son referidas en el Acuerdo de Antidumping como alternativas adecuadas para el cálculo del valor normal. Para asegurar la robustez del análisis, se consideraron varios países al momento de realizar los cálculos. Un ejercicio en esta línea mostraría que en la mayoría de los casos el margen de dumping era negativo, lo que sugiere la posibilidad de que el supuesto dumping se haya diluido o eliminado por completo en los últimos años ${ }^{11}$.

En el análisis de la penúltima resolución de la CDS, se asume que los aumentos en las importaciones o las reducciones en los precios de la RPC de deben al margen del dumping, en la medida que se interpreta como prueba de una continuación del dumping. No se profundiza si ello se debe a las mejores ventajas comparativas del país de origen $u$ a otras razones.

Es relevante mencionar que desde que la RPC accedió a la OMC como economía de mercado, ha habido revisiones periódicas bianuales sobre su grado de adopción de una economía de mercado ${ }^{12}$. En su última revisión, no se reporta que la RPC tenga empresas estatales en la industria de calzado (Organización Mundial del Comercio, 2018). En términos de política industrial planean promocionar industrias intensivas en tecnología como robótica, vehículos con energía no convencional, circuitos, entre otros; mientras tratan de que se reduzca el exceso de capacidad instalada en industrias como la del acero. China está obligada a notificar sus subsidios internos a nivel provincial. De la revisión de estos, no hay ninguno explícito para la industria del calzado, salvo uno sobre industrias livianas en la provincia de Shanghái que expiró en el 2012. El arancel promedio para los calzados es de $11.4 \%$, con una desviación estándar de 6 . No han establecido antidumping o derechos compensatorios a productos de calzado. Las exportaciones de calzado de RPC han sido US\$ 1000 millones anuales en promedio en los últimos años, mientras que sus importaciones alcanzaron US\$ 434 millones $^{13}$.

Por tanto, no pareciera que existan indicios de dumping estratégico ni del estándar por parte de la industria de la RPC.

10 El lesser duty rule o regla del menor derecho, hace referencia a la imposición de derechos antidumping menores al valor del margen de dumping siempre que este valor sea suficiente para remover el daño.

11 De la misma forma, si el valor normal está por debajo del precio de importación, se tendrá un margen de dumping negativo que implicará la inexistencia de la práctica del dumping (APOYO Consultoría, 2016).

12 La más reciente es la de la Organización Mundial del Comercio (2018).

13 Datos extraídos de la Organización Mundial del Comercio y Veritrade. 


\section{Informalidad en mercado}

La relevancia de la informalidad en los mercados en el país se ha tornado recientemente más evidente a raíz de la pandemia del COVID-19. Varias investigaciones, como la de Loayza (2007), han estimado que la informalidad en los mercados de bienes es relativamente alta, en promedio en un $60 \%$.

Si bien no se ha identificado un análisis formal o lineamiento del Indecopi hacia sus órganos resolutivos sobre la informalidad, sí ha habido pronunciamientos institucionales como el siguiente:

Finally, it is important to appoint that we could not deny the importance of informal sector inside the market economy in Peru. In that sense, when a specific anticompetitive conduct is analysed, the authority of competition should not leave out the presence of informal agents, first, when it determines the relevant market and, consequently, the market power that let them cause a major injury for the general economic interest (Organization for Economic Co-operation and Development [OECD], 2009, p. 166) [el énfasis es nuestro $]^{14}$.

La informalidad es relevante en las evaluaciones sobre dumping debido a que afecta la representatividad de la RPN al excluirlos, y porque puede constituir una relevante explicación alternativa al supuesto perjuicio que la RPN ha tenido en los últimos años.

Sin embargo, en el último sunset review de dumping de calzado, la CDS sostuvo que en una revisión de derechos antidumping no era necesario demostrar el nexo causal entre el dumping y el deterioro de la RPN. Ello debido a que la causalidad ya había sido probada previamente por las resoluciones que habían implementado los derechos vigentes.

Asimismo, la CDS resaltó que la informalidad no debería de ser tomada en cuenta en el análisis debido a su carácter ilegal y a la ausencia de información existente de la misma. No obstante, al no considerar estos dos puntos, el análisis económico resulta superficial y no permite delimitar los derechos antidumping efectivamente aplicables ni responder a la cuestión principal del sunset review sobre si las partidas arancelarias analizadas deberían de seguir sujetas a los derechos antidumping. A continuación, se desarrollan ambos puntos y la necesidad de revisarlos a profundidad.

El nexo causal entre las importaciones de un producto específico y su efecto sobre la industria nacional es un punto central dentro del análisis económico. Al momento de implementar derechos antidumping, la autoridad está restringiendo la competencia internacional, como ya se mencionó. Sin perjuicio de lo anterior, estos derechos tienen el objetivo de desincentivar una práctica, mas no de desincentivar la libre competencia entre las partes. No obstante, al evitar el análisis de causalidad, la CDS podría impactar negativamente en el mercado mediante dos canales:

i) que se pasen por alto otros factores que podrían ser la causa del daño o que simplemente ya no exista daño a la RPN debido al supuesto dumping ${ }^{15} ; \mathrm{y}$,

ii) generar barreras que obstaculizan la libre competencia.

De esta forma, para evitar la persistencia de un problema no observado y para fomentar los beneficios que trae consigo la libre competencia, el análisis de causalidad debería ser un requisito para las revisiones de derechos antidumping. Los efectos negativos que pueden resultar de evitar el mencionado análisis son evidentes. No obstante, por criterio de la CDS, se pasó por alto la necesidad de incluir a la informalidad en el mercado como una de las causas por las cuales una industria nacional se puede deteriorar en el tiempo.

En este contexto, es pertinente preguntarse si la informalidad debería ser descartada del análisis solo por ser una práctica ilícita, y si, en efecto, no existe data disponible para poder analizarla. Con respecto al primer punto, es importante señalar que la informalidad tal como se mencionó es un factor relevante en buena parte de los mercados de bienes peruano. Esto se puede observar también al analizar la tasa de informalidad del empleo a nivel agregado que, en el 2018, representaba el $72.4 \%$

14 Por último, es importante señalar que no es posible ignorar la relevancia del sector informal en la economía de mercado peruana. En ese sentido, cuando se analiza una conducta anticompetitiva específica, la autoridad de la competencia no debe dejar de lado la presencia de agentes informales, en primer lugar, cuando se determina el mercado de referencia y, en consecuencia, el poder de mercado que les permite causar una lesión grave para el interés económico general [traducción libre] [el énfasis es nuestro].

15 Otros factores que podrían estar afectando a la supuesta continuación del daño incluyen también la dinámica de empleo y salarios del mercado laboral, la capacidad empleada, el valor de las importaciones de los insumos y bienes de capital de la industria, y las exportaciones de la RPN. 
de todos los trabajadores a nivel nacional (Instituto Nacional de Estadística e Informática [INEI], 2019). Como consecuencia, el análisis de los mercados relevantes debería de tomar en consideración la efectiva competencia que las empresas formales tienen por parte de su contraparte informal.

En segundo lugar, los criterios en análisis de dumping no son limitativos. Dentro del propio acuerdo de Antidumping de la OMC, se estipula que la autoridad competente de analizar y tomar decisiones en casos de dumping tiene la facultad para utilizar otros elementos externos si es que ellos permiten un análisis integral del caso. Como se ha descrito, la informalidad en el Perú calza dentro de este marco. Por último, encuestas oficiales como la Encuesta Nacional de Hogares (ENAHO) y el trabajo conjunto de diversas entidades del Estado, permiten poder estimar la cantidad de individuos que se desenvuelven en el sector formal e informal de distintas industrias.

\section{El efecto renta por la Zofratacna}

La Zona Franca de Tacna o Zofratacna, es un espacio geográfico dentro del Perú que goza de beneficios y excepciones tributarias y arancelarias que permiten la importación de bienes a un menor costo. En esta línea, y como señala la CDS en el Documento de Hechos Esenciales (DHE) reportado en el 2016, los bienes importados hacia la Zofratacna no pagan derechos antidumping.

Ello permitiría un arbitraje regulatorio causado por la exoneración de los derechos antidumping para los productos que son importados hacia y desde la Zofratacna. Es decir, una empresa pueda importar productos del extranjero a la Zofratacna y, luego, exportarlos hacia el Perú. De este modo, una empresa podría vender productos que no incorporan este costo obteniendo un mayor margen de ganancia $u$ ofreciendo menores precios que la competencia.

Es relevante destacar que Zofratacna, pasó a constituir el principal o segundo exportador de los bienes sujetos a antidumping en el periodo 2011 2014 , alcanzando el $27 \%$ del mercado del producto investigado (Resolución 0281-2018/SDC-INDECO$\mathrm{PI}, 2018)$.

Ello implica que las empresas operando en Zofratacna gocen de una protección efectiva sumamente alta, debido a que están desgravados en las importaciones de los insumos para calzado, y a que sus exportaciones de calzado hacia el país no paga derechos compensatorios. Ello muestra un fuerte incentivo a incurrir en mercantilismo.

En términos sencillos, ese nivel de protección efectiva implica que la RPN puede aumentar hasta el doble de su valor agregado solo debido a la protección comercial. El valor agregado es la suma del retorno al capital (utilidades, depreciación más intereses), más el retorno al factor trabajo (remuneraciones) y el impuesto a la renta de la producción nacional. Potencialmente, la RPN puede casi duplicar sus rentas gracias a la protección comercial. Otra manera de interpretarlo es que la RPN de la Zofratacna podría tener el doble de mayores costos y aún poder competir.

La posición de la CDS en las pasadas resoluciones ha sido que este efecto no es de su competencia funcional; no obstante, desde la perspectiva de libre competencia, claramente es relevante.

\section{RECOMENDACIONES}

Se sugieren las siguientes recomendaciones para la mejor aplicación de las políticas de dumping y de libre competencia.

1. En aplicación de la función que tiene el propio Indecopi para mejorar la competencia mediante recomendaciones a entidades públicas, es posible identificar tres grandes opciones:

i) denunciar al Acuerdo Antidumping de la OMC;

ii) traspasar las competencias sobre dumping y subvenciones a otra entidad del Estado como el Ministerio de Economía y Finanzas o el Ministerio de Comercio Exterior y Turismo; y,

iii) aplicar la metodología de análisis de libre competencia a los casos de dumping.

La primera no es viable al no justificar las consecuencias negativas que tendría en otros ámbitos. La segunda no resolvería el problema de fondo. Por ello, lo recomendable es la tercera opción ${ }^{16}$. Una ventaja de ello es precisamente que ambas funciones están bajo la competencia de una misma entidad.

2. Los casos de dumping que debería conocer la autoridad deben ser solo aquellos que caen dentro de las categorías de dumping

16 Gonzáles de Cossío (2006) propone también este enfoque para el caso mexicano. 
estratégico y de dumping predatorio. Los otros deberían ser desestimados. Dado que el acuerdo de la OMC no incluye criterios limitativos, se considera que ello es viable dentro de la discrecionalidad que tiene la autoridad del país.

3. Los análisis de los casos de dumping deben incorporar el análisis de mercado relevante, la posición de dominio, y los efectos de la práctica sobre productores y consumidores con un empleo de escenarios contrafactuales con indicadores complementarios a los listados por el acuerdo de la OMC y la reglamentación nacional.

4. El margen de dumping debería ser estimado y/o actualizado en todos los procedimientos, sean estos provisionales, definitivos, hechos esenciales, o de expiración de medida, con alguno de los métodos contemplados en el Acuerdo Antidumping de la OMC.

5. La falta de participación de las empresas investigadas o la falta de información no debe justificar decisiones que corten camino con atajos, que perjudiquen en última instancia a los consumidores.

6. Se debe tratar de evitar que los casos investigados estén motivados para capturar rentas, o que promuevan acuerdos colusorios.

7. La prospectiva que se hacen en los procedimientos de expiración de medidas debería basarse en análisis de escenarios posibles que incluyan las variables relevantes según las técnicas de la especialidad de prospectiva.

8. Se sugiere que se apruebe un lineamiento sobre el tratamiento de la informalidad para los órganos resolutivos del Indecopi.

\section{REFERENCIAS}

Anderson, J. E. (1994). Strategic Lobbying and Antidumping. Journal of Economic Integration, 9(2), 129-155.

APOYO Consultoría (2016). Comentarios a la Resolución № 047-2016/CDB-INDECOPI.

Centro de Investigación de la Universidad del Pacífico (1997). Efectos de la Existencia de Dumping Sobre los Sectores Manufactureros de Prendas de Vestir y Calzado Peruanos.

González de Cossío, F. (2006) Discriminación de precios, dumping y depredación. Tres discipli- nas, un fenómeno económico. Boletín mexicano de derecho comparado, 39(115), 125-146.

Instituto de Defensa de la Competencia y Propiedad Intelectual [Indecopi] (2020). Propuestas relativas al fortalecimiento institucional del Indecopi (Documento de Trabajo Institucional 003-2020). https://www.indecopi.gob. pe/documents/1902049/3994339/RES038DOCUMENTO+DE+TRABAJO+INSTITUCION $A L+003-2020$. pdf/1ae0fed3-685c-4a3b-a173$87 a d 6 d 96303 c$

Instituto Nacional de Estadística e Informática [INEI] (2019). Producción y Empleo Informal en el Perú. Cuenta Satélite de la Economía Informal 2007-2018. https://www.inei.gob.pe/media/MenuRecursivo/publicaciones_digitales/ Est/Lib1701/libro.pdf

Kafka, F. (1997). Teoría Económica. Universidad del Pacífico.

Krueger, A. O. (1974). The Political Economy of the Rent-Seeking Society. American Economic Review, 64(3), 291-303.

Loayza, N. (2007). The causes and consequences of informality in Peru. Banco Central de Reserva del Perú.

Mai, Y. (2002). An analysis of EU antidumping cases against China. Asia-Pacific Development Journal, 9(2), 131-150.

Organización Mundial del Comercio (2001). Adhesión de la República Popular de China (WT/L/432). https://docs.wto.org/dol2fe/Pages/SS/directdoc.aspx?filename=S:/WT/L/432. pdf\&Open=True

(2018). Trade policy review. Report by the secretariat. China. WTO. WT/TPR/S/375. https:// www.wto.org/english/tratop_e/tpr_e/ s375_e.pdf

Organización para la Cooperación y el Desarrollo Económicos [OECD] (2009). Peru Competition Policy and the Informal Economy. Policy Roundtables. https://www.oecd.org/daf/competition/44547855.pdf

(2018) Exámenes inter-pares de la OCDE y el BID sobre el derecho y política de competencia: Perú. https://www.oecd.org/daf/competition/ examenes-inter-pares-ocde-bid-derecho-y-politica-del-competencia-peru-2018.htm 
Pierce, R. J. (2000). Antidumping law as a means of facilitating cartelization. Antitrust Law Journal, $67(3), 725-743$

Secretaría Técnica de la Comisión de Dumping, Subsidios y Eliminación de Barreras Comerciales No Arancelarias del Indecopi (1997). Informe 005-97-INDECOPI/CDS (Perú).

(2016). Informe 062-2016/CDB-INDECOPI.

Sykes, A. O., \& Cooper, R. N. (1998). Antidumping and Antitrust: What Problems Does Each Address? Brookings Trade Forum, 1-53.

Tullock, G. (1967). The Welfare Costs of Tariffs, Monopolies and Theft. Western Economic Journal, 5(3), 224-232. https://doi. org/10.1111/j.1465-7295.1967.tb01923.x

Voon, T. (2007). Review of Edwin Vermulst, the WTO Anti-Dumping Agreement: A Commentary. International and Comparative Law Quarterly, 56(2), 463-470.

Willig, R. (1998). Economic Effects of Antidumping Policy. Brookings Trade Forum, 57-79.

\section{LEGISLACIÓN, JURISPRUDENCIA Y OTROS DOCU- MENTOS LEGALES}

Acuerdo General sobre Aranceles Aduaneros y Comercio de 1947 (GATT de 1947), 30 de octubre de 1947. https://www.wto.org/spanish/ docs_s/legal_s/06-gatt_s.htm

Acuerdo General sobre Aranceles Aduaneros y Comercio de 1994 (GATT de 1994), 15 de abril de 1994. https://www.wto.org/spanish/docs_s/ legal_s/06-gatt_s.htm

Acuerdo relativo a la Aplicación del Artículo VI del GATT de 1994 de la Organización Mundial del Comercio (Acuerdo Antidumping de la OMC), 15 de abril de 1994.

Comisión de Dumping, Subsidios y Eliminación de Barreras Comerciales No Arancelarias, 15 de marzo de 1996, Resolución 002-96/CDS-INDECOPI (Perú)

Comisión de Dumping, Subsidios y Eliminación de Barreras Comerciales No Arancelarias, 7 de marzo de 1997, Resolución 005-97-INDECOPI/ CDS (Perú).

Comisión de Dumping, Subsidios y Eliminación de Barreras Comerciales No Arancelarias del Indecopi, 1 de abril 2016, Resolución 047 -
2016/CDB-INDECOPI, Expediente 007-2014/ CFD (Perú).

Comisión de Dumping, Subsidios y Eliminación de Barreras Comerciales No Arancelarias del Indecopi, 4 de abril de 2019, Resolución 046-2019-CDB-INDECOPI, Expediente 0072014/CFD (Perú).

Comisión de Fiscalización de Dumping y Subsidios del Indecopi, 8 de marzo de 1996, Resolución 001-96-INDECOPI/CDS (Perú).

Comisión de Fiscalización de Dumping y Subsidios del Indecopi, 21 de enero de 2000, Resolución 001-2000/CDS-INDECOPI (Perú).

Comisión de Fiscalización de Dumping y Subsidios del Indecopi, 19 de septiembre de 2005, Resolución 122-2005/CDS-INDECOPI (Perú).

Comisión de Fiscalización de Dumping y Subsidios del Indecopi, 5 de noviembre de 2009, Resolución 181-2008/CFD-INDECOPI (Perú).

Decreto Legislativo 807, Facultades, normas y organización del Indecopi, Diario Oficial El Peruano, 18 de abril de 1997 (Perú).

Decreto Legislativo 1205, Decreto Legislativo que modifica el Decreto Legislativo 1034, que aprueba la Ley de Represión de Conductas Anticompetitivas, Diario Oficial El Peruano, 23 de septiembre de 2015 (Perú).

Decreto Supremo 006-2003-PCM, Reglamentan normas previstas en el "Acuerdo Relativo a la Aplicación del Artículo VI del Acuerdo General sobre Aranceles Aduaneros y Comercio de 1994", el "Acuerdo sobre Subvenciones y Medidas Compensatorias" y en el "Acuerdo sobre Agricultura", Diario Oficial El Peruano, 11 de enero de 2003 (Perú)

Decreto Supremo 043-97-EF, Reglamento sobre Dumping y Subvenciones, Diario Oficial El Peruano, 29 de abril de 1997 (Perú).

Decreto Supremo 133-91-EF, Reglamento sobre Dumping y Subvenciones, Reemplaza y deroga el Decreto Supremo 043-97-EF y sus normas, modificatorias, Diario Oficial El Peruano, 11 de enero de 2003 (Perú).

Decreto Supremo 144-2000-EF, Modifican el Reglamento sobre Dumping y Subvenciones, Diario Oficial El Peruano, 23 de diciembre de 2000 (Perú). 
Decreto Supremo 225-2001-EF, Modifican el Decreto Supremo 043-97-EF, mediante el cual se establecieron normas para evitar y corregir distorsiones de la competencia en el mercado generadas por el dumping y las subvenciones, Diario Oficial El Peruano, 5 de diciembre de 2001.

Presidencia del Consejo Directivo del Indecopi, 28 de febrero de 2020, Resolución 038-2020-INDECOPI/COD (Perú).

Resolución Legislativa 26407, Aprueban Acuerdo por el que se establece la Organización Mundial del Comercio y los Acuerdos Multilaterales
Contenidos en el Acta Final de la Ronda de Uruguay, Diario Oficial El Peruano, 18 de diciembre 1994 (Perú).

Sala Especializada en Defensa de la Libre Competencia del Tribunal de Defensa de la Competencia y de la Propiedad Intelectual.

Sala Especializada en Defensa de la Libre Competencia del Tribunal de Defensa de la Competencia y de la Protección de la Propiedad Intelectual, 14 de febrero de 2019, Resolución 02822018/SDC-INDECOPI, Expediente 049-2014/ CFD (Perú). 